\title{
Weis-Fogh Mechanism Mathematic Model of Wave Power Generation Device
}

\author{
Xin Chen, Quan Kuang, Yang Li, Songbo Wang, Yunling Ye, Shesheng Zhang* \\ Wuhan University of Technology, Wuhan, China \\ Email: sheshengz@qq.com
}

Received 4 October 2014; revised 4 November 2014; accepted 4 December 2014

Copyright (C) 2014 by authors and Scientific Research Publishing Inc.

This work is licensed under the Creative Commons Attribution International License (CC BY). http://creativecommons.org/licenses/by/4.0/

c) (i) Open Access

\begin{abstract}
A Weis-Fogh mechanism wave power generation system is designed, its physical model and mathematical model are discussed, and the component expressions of fluid dynamic expression are derived. Adopting numerical integral algorithm, the work done by fluid force acting on wing is calculated.
\end{abstract}

Keywords

Wave, Weis-Fogh, Power Generation, Mathematical Model

\section{Introduction}

Saving energy and reducing contaminant emission are important researching projects for economics and human living environment. As we know, using wave energy to generate electricity can reduce oil and coal consumption, and decease dust and emissions of harmful substances emission. So it attracts scientists to carry out power generation of scientific research by using wave energy [1]. According to the statistics data, there is huge amount of wave energy stored in the ocean, which can be used to transfer to electricity energy [2]. There are many devices to absorb wave energy, such as floating rope round of absorbing wave energy device [3], matrix of relative movement absorbing wave energy device [4], buoy type absorption wave energy device [5] and floating type absorption wave energy device [6], according to the fluctuation characteristics of waves. All of those devices didn't use the theory of Weis-Fogh [7]. According to the fluid mechanics theory of Weis-Fogh mechanism [8], Weis-Fogh mechanism is a simulation of the wasp flying $\mathrm{V}$ vibration device, which is different from conventional produce wing lift mechanism [9]. Rigid body test measures the lift coefficient as high as $7-8$ when the wing opens [10] [11]. Thus, Weis-Fogh mechanism is possible to be used in the case of wave energy power generation. But it needs a lot of scientific research.

"Corresponding author.

How to cite this paper: Chen, X., Kuang, Q., Li, Y., Wang, S.B., Ye, Y.L. and Zhang, S.S. (2014) Weis-Fogh Mechanism Mathematic Model of Wave Power Generation Device. Open Journal of Fluid Dynamics, 4, 373-378.

http://dx.doi.org/10.4236/ojfd.2014.44028 
The paper will consider Weis-Fogh wave power generation device, calculating its fluid doing work acted on the wing.

\section{The Working Principle of the Generation Set}

Weis-Fogh wave power energy generation device makes of two parts mainly: one is Weis-Fogh absorbing wave energy mechanism; another is the power generation mechanism. As Figure 1 shows, Weis-Fogh absorbing wave energy mechanism is below water surface, others are power generation mechanism. Those two parts are connected with push-rods which make the power generation device and Weis-Fogh absorbing wave energy mechanism as an integral device. One endpoint of the push-rod is connected with wing $\mathrm{AB}$, the other point is connected with power generation. In Figure 1, wave makes the wing AB moving up and down, and wing AB drives the push-rod moving up and down at same times. As this way, the power generation device transfers kinetic energy into electrical energy.

\subsection{Weis-Fogh Wave Power Device}

The wing AB's movements of Weis-Fogh mechanism wave power device will be described as below paper. According to Figure 2, at the beginning, the wing attaches the low wall tightly. Acted by the wave force, the endpoint of wing B leaves the wall, the wing opens, around the A point rotation. Such movement is called as opening stage, see Figure 2(a). After the angle is opened to the $\beta$, the point A leaved away from bottom wall, and all point of wing $\mathrm{AB}$ moves up in same speed until the point $\mathrm{B}$ meets the top wall. In this case, wing $\mathrm{AB}$ moves up without angle turning, see Figure 2(b). After point B attaches top wall, the wing makes close motion until the point A meets the top wall, such movements is called the closing stage, see Figure 2(c). As the same way, acted by wave force, the point A leaves the wall, the wing opens, around the B point rotation. Such movement is also called as opening stage. After the angle is opened to the $\beta$, the point $\mathrm{B}$ leaved away from bottom wall, and all point of wing $\mathrm{AB}$ moves down in same speed until the point $\mathrm{A}$ meets the low wall. In this case, wing $\mathrm{AB}$ moves up without angle turning. After point $\mathrm{A}$ attaches low wall, the wing makes close motion until the point B meets the low wall, Such movements is also called the closing stage. In such way, the wing finishes one cycle movement.

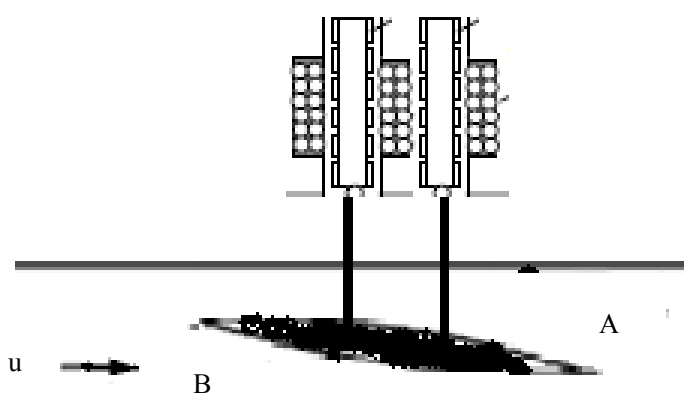

Figure 1. Weis-Fogh wave power generation device.

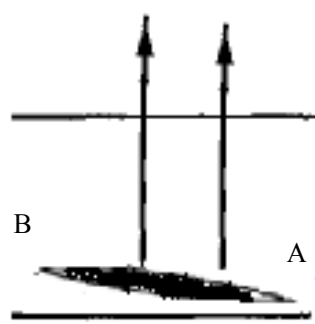

(a)

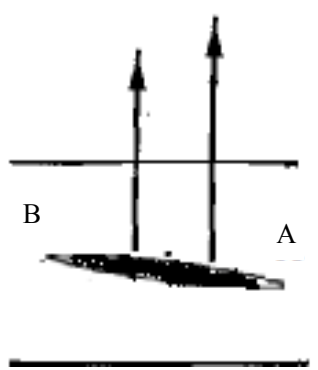

(b)

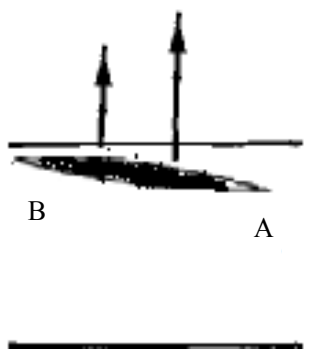

(c)

Figure 2. The movements of the Weis-Fogh wave power generation device. 


\subsection{Power Generation Device}

The power generation device is shown as Figure 1, it connected with Weis-Fogh absorbing wave energy mechanism by push-rod, and transports wave energy into electrical energy. There are two push-rods by which can control the wing rotation angle.

\subsection{Force Model}

According to the Weis-Fogh wave energy device, the wing is acted by fluid force. During the opening stage, the endpoint of wing A doesn't leave the wall, the fluid force acting on wing can be calculated by using the theory of Weis-Fogh mechanism. Between the opening stage and the closed stage, the fluid force acting on wing can be calculated by fluid-solid coupling method. At the opening stage and at the closing stage, the fluid is supposed as ideal.

\subsection{The Equations of Motion of the Wing}

During the opening stage, the low wall supports wing at point A, the push-rod thrusts wing, and fluid force acts on the wing. As the influence of above forces, the wing rotates around the endpoint of wing A, the motion equation is:

$$
J \pi \ddot{\alpha}=M_{f}-M_{L}
$$

where $J$ is the moment of inertia, $M_{f}$ is the torque acted by fluid force on the wing, $M_{L}$ is the torque which the push-rod acts on the wing. Let opening angle is $\left(\pi \alpha_{0}\right)$ and the velocity is $\Omega_{0}$ when $T=0$.

Above equation is also used to the closing stage.

Between the opening stage and the closing stage, the wing just moves up and down, the corresponding equation of motion is:

$$
m \ddot{y}=F_{y}-F_{L}
$$

Here $m$ is mass of wing, $F_{y}$ is the force of the fluid to the wing projected on the $y$ direction, $F_{L}$ is the push from push-rod. When $T=0$, let $y$ equal to $y_{0}$, velocity is $V_{y_{0}}$.

This paper will consider the fluid power during opening stage and closing stage.

\section{Fluid Power at Opening and Closing Stage}

During this period, the wing's thickness is considered very thin compared with its length. For using theory of Weis-Fogh mechanism, the wing can be supposed as a tablet. As Figure 3(a) shows, the fluid domain surrounded by boundary $\mathrm{GEFA}_{1} \mathrm{BA}_{2} \mathrm{FG}$ is mapped into up $\zeta$ plane. The boundary point $G$ on physic plane mappings to the real axis point $\xi=(-\infty)$ on mapping plane. Point $E$ mappings to point $\xi=b$, point $A_{1}$ mappings to point $\xi=-1$, point $B$ mappings to point $\xi=a$, point $A_{2}$ mappings to point $\xi=1$, point $F$ mappings to point $\xi=(+\infty)$.

By conformal mapping of complex function theory, the physical to the mapping transformation formula for plane area is [7]:

$$
\begin{aligned}
& z=K h(\xi) \mathrm{e}^{\alpha \pi i} \\
& h(\xi)=\int_{-1}^{\xi} h^{\prime}(t) \mathrm{d} t
\end{aligned}
$$

where $K$ is the transform constant, function $h^{\prime}$ is expressed as:

$$
h^{\prime}(t)=(t-b)^{-1}(t+1)^{-\alpha}(a-t)(1-t)^{\alpha-1}
$$

where $K, a, b$ are constant and related to these expressions below:

$$
\begin{aligned}
& C=K h(a), \quad d=K \pi, \\
& a=b-(b-1)^{1-\alpha}(1+b)^{\alpha} .
\end{aligned}
$$



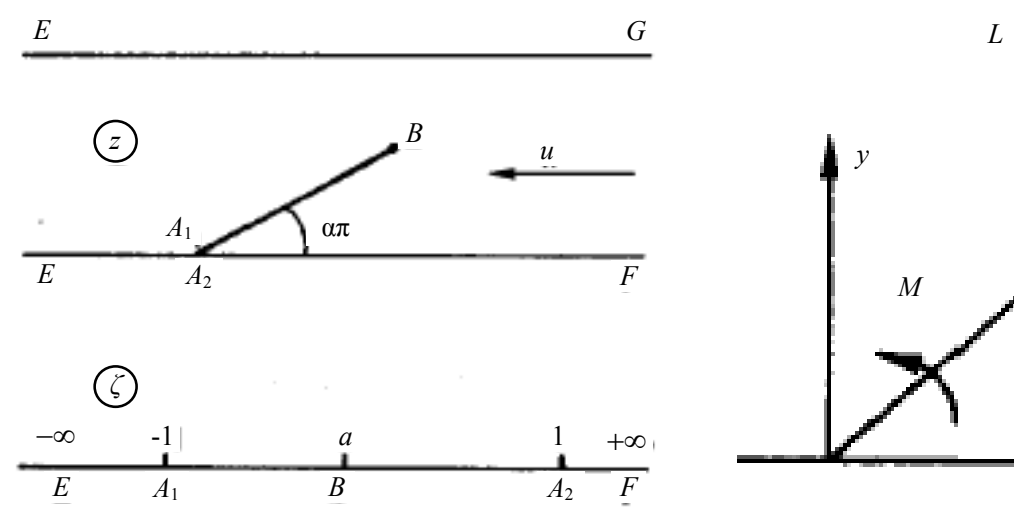

(a)

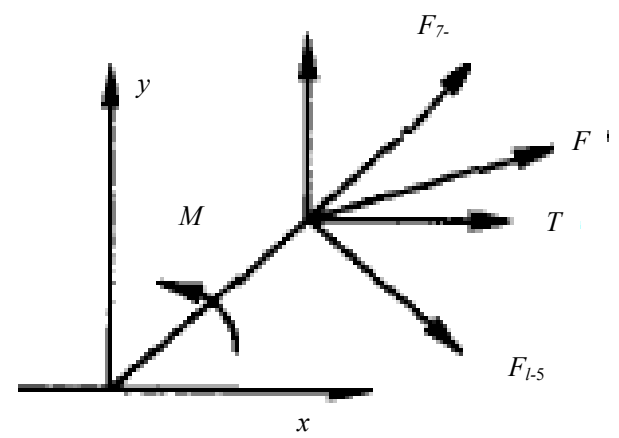

(b)

Figure 3. (a) The coorespondings between physic plane and mapping plane; (b) Force direction.

Here $C$ is the length of wing, $d$ is grid spacing. The steps of calculation is: getting the constant $K$ firstly, then solve the constant $a$, finally calculating the constant $b$.

The complex potential is:

$$
\begin{aligned}
& W=\Omega G-K u \ln (\zeta-b), \\
& G=\frac{K^{2}}{2 \pi} \int_{-1}^{1} \frac{h^{2}}{\zeta-t} \mathrm{~d} t .
\end{aligned}
$$

Here $\Omega$ is rotating angular velocity, $u$ is the flow speed. According Bernoulli equation, we have:

$$
p=p-\rho(\operatorname{Re} W)_{t}^{\prime}-0.5 \rho\left|W_{\zeta} h^{\prime}\left(\zeta^{\prime}\right)\right|^{2}
$$

Integral the pressure along the wing surface, we get the force acting on the wing:

$$
i \frac{F}{\rho} \mathrm{e}^{-\alpha \pi i}=\Omega^{\prime} F_{1}-u^{\prime} F_{3}+\Omega^{2} F_{2}-\Omega u F_{4}+u^{2} F_{5}+i\left(\Omega^{2} F_{7}-\Omega u F_{8}+u^{2} F_{9}\right)
$$

where:

$$
\begin{gathered}
F_{1}=-\operatorname{Re} \int_{-1}^{1} G K h^{\prime} \mathrm{d} t, \quad F_{3}=-\operatorname{Re} K \int_{-1}^{1} \ln (t-b) K h^{\prime} \mathrm{d} t, \\
F_{22}=-0.5 \int_{-1}^{1} G_{\zeta}^{2}\left|z_{\zeta}^{\prime}\right|^{-2} K h^{\prime} \mathrm{d} t, \quad F_{42}=-K \int_{-1}^{1} \frac{G_{\zeta}}{t-b}\left|z_{\zeta}^{\prime}\right|^{-2} K h^{\prime} \mathrm{d} t, \\
F_{52}=-0.5 K \int_{-1}^{1}\left|(t-b) z_{\zeta}^{\prime}\right|^{-2} K h^{\prime} \mathrm{d} t . \\
F_{2}=\operatorname{Re} F_{42}+\frac{F_{1 a}}{\pi}, \quad F_{7}=\operatorname{Im} F_{22}, \\
F_{4}=\operatorname{Re} F_{42}+\frac{F_{3 a}}{\pi}, \quad F_{8}=\operatorname{Im} F_{42}, \\
F_{4}=\operatorname{Re} F_{52}, \quad F_{9}=\operatorname{Im} F_{52} .
\end{gathered}
$$

Here $\left(-F_{7}\right),\left(-F_{8}\right),\left(-F_{9}\right)$ are frontal appeal, leading from the wing tips to the root, shown as Figure 3(b).

The force mapping on $x$ axis calls push.

$$
T=\Omega^{\prime} T_{1}-u^{\prime} T_{3}+\Omega^{2} T_{2}-\Omega u T_{4}+u^{2} T_{5}
$$


where

$$
\begin{aligned}
& T_{1}=F_{1} \sin (\alpha \pi), \quad T_{3}=F_{3} \sin (\alpha \pi), \quad T_{5}=0, \\
& T_{2}=F_{2} \sin (\alpha \pi)+F_{7} \cos (\alpha \pi), \\
& T_{4}=F_{4} \sin (\alpha \pi)+F_{8} \cos (\alpha \pi) .
\end{aligned}
$$

The force mapping on $y$ axis calls lift

$$
L=\Omega^{\prime} L_{1}-u^{\prime} L_{3}+\Omega^{2} L_{2}-\Omega u L_{4}+u^{2} L_{5}
$$

where

$$
\begin{aligned}
& L_{1}=-F_{1} \cos (\alpha \pi), \quad L_{3}=-F_{3} \cos (\alpha \pi), \\
& L_{2}=-F_{2} \cos (\alpha \pi)+F_{7} \sin (\alpha \pi), \\
& L_{4}=-F_{4} \cos (\alpha \pi)+F_{8} \sin (\alpha \pi), \\
& L_{5}=-F_{5} \cos (\alpha \pi)+F_{9} \sin (\alpha \pi) .
\end{aligned}
$$

correspondingly, the moment is

$$
\frac{M}{\rho}=\Omega^{\prime} M_{1}-u^{\prime} M_{3}+\Omega^{2} M_{2}-\Omega u M_{4}+u^{2} M_{5}
$$

where

$$
\begin{aligned}
& M_{1}=\operatorname{Re} \int_{-1}^{1} G K h h^{\prime} \mathrm{d} t, \quad M_{3}=\operatorname{Re} \int_{-1}^{1} \ln (t-b) K^{3} H h^{\prime} \mathrm{d} t, \\
& M_{22}=0.5 K \int_{-1}^{1} G_{R}^{2} \frac{h}{h^{\prime}} \mathrm{d} t, \quad M_{42}=0.5 K \int_{-1}^{1} \frac{G_{\alpha}}{t-b} \frac{h}{h^{\prime}} \mathrm{d} t, \\
& M_{5}=0.5 K \int_{-1}^{1}(t-b)^{-2} \frac{h}{h^{\prime}} \mathrm{d} t, \\
& M_{2}=M_{22}+\frac{\partial M_{1}}{\pi \partial \alpha}, \quad M_{4}=M_{42}+\frac{\partial M_{3}}{\pi \partial \alpha} .
\end{aligned}
$$

Integration of the moment along the angle is the work of the wing

$$
\begin{aligned}
& \frac{W}{\rho}=\Omega^{\prime} W_{1}-u^{\prime} W_{3}+\Omega^{2} W_{2}-\Omega u W_{4}+\mathrm{u}^{2} W_{5}, \\
& W_{J}=\int_{0}^{\beta} M_{J} \mathrm{~d}(\alpha \pi), \quad j=1,2,3,4,5 .
\end{aligned}
$$

\section{The Calculation of Fluid Power}

According the above equations, the code is written to calculate the fluid power. Figure 4(a) shows that the components of force acting on wing varied with the angles when the grid spacing and the wing chord length ratio is 0.5 . The curves' widths vary large with the component of the subscript. When the dimensionless angle $\alpha$ approaches to $0.5, F_{1}$ and $F_{2}$ tend to infinity, $F_{4}$ and $F_{5}$ tend to $0, F_{7-9}$ tend to constants.

When the grid spacing and the wing chord length ratio is 0.5, Figure 4(b) shows the component's moment varied with the angles. The curves' widths vary large with the component of the subscript. The horizontal axis is dimensionless angle $\alpha=\beta / \pi$. When the dimensionless angle $\alpha$ approaches to 0.5 , the moment $W_{2}$ tends to infinity, and the moment $W_{1-3}$ and $F_{3}$ tend to constants.

\section{Conclusion}

The fluid force acting on the wing is the most important for Weis-Fogh wave power generation device. It is 


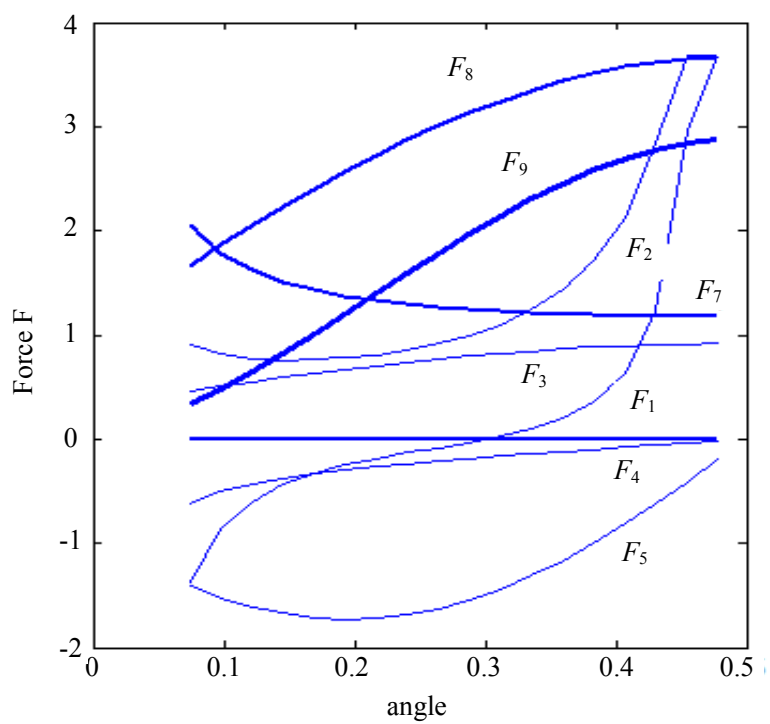

(a)

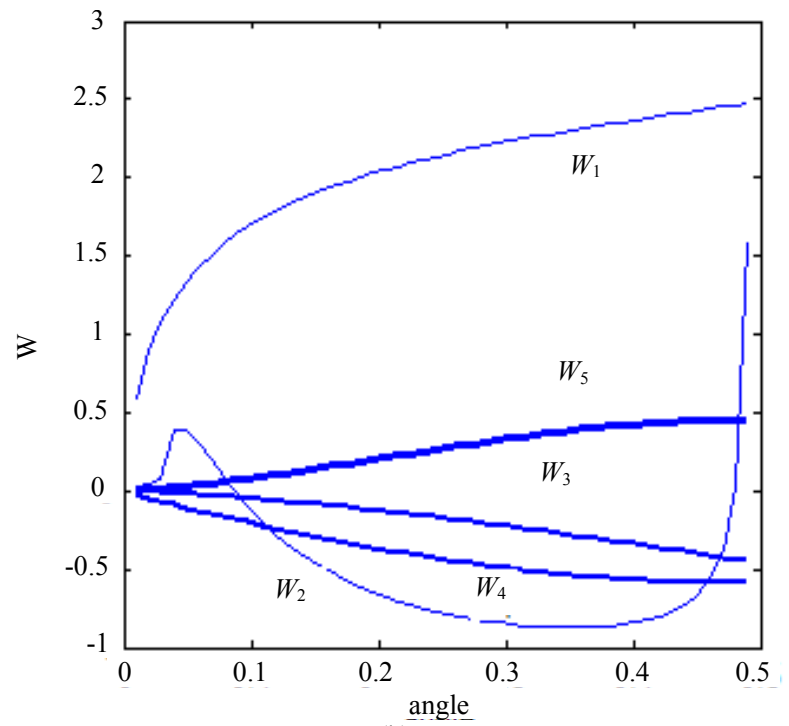

(b)

Figure 4. (a) Component varied with the angle; (b) The component moments varied with the angle.

always calculated before device designed. According to the Weis-Fogh mechanism theory and conformal transformation theory, the fluid dynamics model of double push-rod cascade wave power generation device is built, and at opening stage and close stage, the expression formula of work done by fluid force acting on wing is derived by using integral method. Such formula is dependent with coming velocity $u$ and wing turning velocity, and can be calculated and saved in the disk. It can increase the speed of calculating fluid force and work.

\section{Acknowledgements}

The paper is financially supported by China National Science \& Technology Innovation Fund (No. 20141049705002).

\section{References}

[1] Kang, Q., Xiao, X., Lie, Z.X. and Huang, L.P. (2013) Control Strategy Optimization of Direct Drive Power Output Type Generation System. Automation of Electric Power Systems, 3, 24-29.

[2] Jiao, Y.F. and Liu, Y.L. (2010) The Current Status and Prospect of Wave Power Generation. Chinese High-Tech Enterprises, 12, 89-90.

[3] Qu, Y.M. (2011) Floating Sheave Wave Power Generation Technology. Rural Electrification, 6, 56-57.

[4] Wang, K., Shen, R.H. and Wen, H.A. (2014) A Wave Power Generation-Wave Energy Matrix of Relative Motion Unit Mechanism. Modern Machinery, 2, 40-42.

[5] Wang, S.C., Che, R.W., Hu, C.S., Zhang, Y.H. and You, W. (2007) Research on a Float Type Wave Power Generation Mechanism. Journal of Solar Energy, 11, 1266-1271.

[6] Li, D., Bai, B.D., Yu, Q. and Zhu, B.F. (2011) Research on Floating Type of Wave Power Generation System. Journal of Solar Energy, 10, 1566-1569.

[7] Zhang, S.S., et al. (1996) Hydrodynamic Model of Weis-Fogh Mechanism Hydrofoil. Journal of Hydrodynamics, 4, 35-39.

[8] Zhang, S.S., et al. (1999) Research and Progress of Weis-Fogh Mechanism Hydrodynamics. Journal of Hydrodynamics, 3, 55-60.

[9] Tsutahara, M. and Kimura, T. (1987) An Application of the Weis-Fogh Mechanism to Ship Propulsion. Journal of Fluid Engineering, 109, 107-113. http://dx.doi.org/10.1115/1.3242629

[10] Weis-Fogh, T. (1973) Quick Estimates of Flight Fitness in Hovering Animals Including Novel Mechanism for Lift Production. The Journal of Experimental Biology, 59, 169-230.

[11] Maxworthy, T. (1979) Experiments on the Weis-Fogh Mechanism of Lift Generation by Insects in Hovering Flight. Part 1. Dynamics of the "Fling". Journal of Fluid Mechanics, 93, 47-63. http://dx.doi.org/10.1017/S0022112079001774 
Scientific Research Publishing (SCIRP) is one of the largest Open Access journal publishers. It is currently publishing more than 200 open access, online, peer-reviewed journals covering a wide range of academic disciplines. SCIRP serves the worldwide academic communities and contributes to the progress and application of science with its publication.

Other selected journals from SCIRP are listed as below. Submit your manuscript to us via either submit@scirp.org or Online Submission Portal.
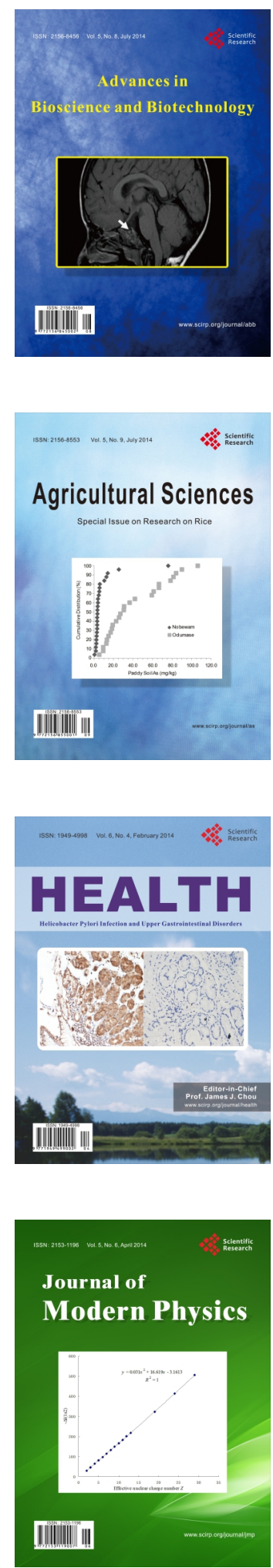
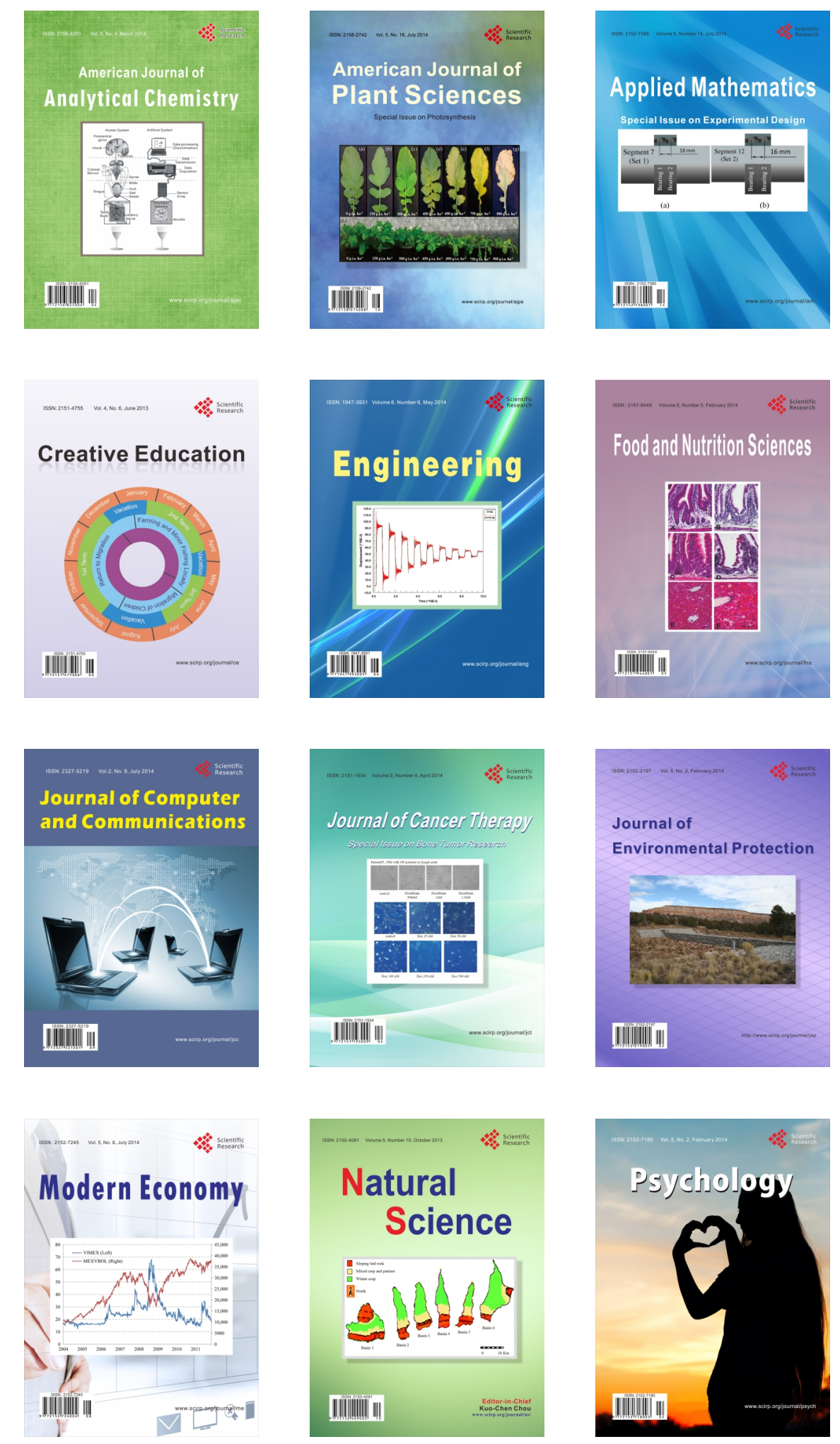